\title{
Conocimientos, actitudes y prácticas acerca del VIH/SIDA en jóvenes de nivel medio de Educación, del área metropolitana, Paraguay
}

\author{
ML MACCHI ${ }^{1}$, BENÍTEZ LEITE S. ${ }^{1}$, CORVALÁN A. ${ }^{2}$, NUÑEZ C. ${ }^{3}$, ORTIGOZA D. ${ }^{1}$ \\ 1. Cátedra de Pediatría, Centro Materno Infantil. Facultad de Medicina U.N.A. \\ 2. Estudiante de nivel Universitario. Derecho U.N.A. \\ 3. Instructor Medicina de la Comunidad. Facultad de Medicina U.C.A.
}

\begin{abstract}
Knowledge, attitudes, and behavior related to HIV/AIDS in high-school aged youth in the Metropolitan Area of Asunción, Paraguay

Introduction: The current generation of youths is the largest in history; almost half of the world's population are less than 25 years of age. They have never known a worldwithout AIDS. The few countries that have achieved a reduction in the prevalence of HIV have done so above all by inducing young people to practice safe behavior. Objectives: Determine the HIV/AIDS related knowledge, attitudes, and practices of high-school aged students in public and private secondary schools of the cities of Asuncion and Lambare, Paraguay. Material and Methods: The study is prospective, analytical, cross-sectional, and observational. A self-administered voluntary survey was given to students of the 1st-, 2d-, and 3d-year students (corresponding to grades 10,11, and 12) of the mean of the high schools in Asuncion and Lambare during the month of August, 2006. Results: A total of 336 surveys were analyzed; the mean age of respondents was 16 years-of-age, and 48 percent were female. The source of information cited most often by respondents, and the one they rated most important, was their school. Of the respondents, $42 \%$ reported having sexual relations, including $56.5 \%$ of the males and $27 \%$ of the females. The mean age of first intercourse was 14.6 years, with $72 \%$ of the males reporting that they first experienced intercourse before 15 years of age. Overall, 55\% reported using prophylactics during casual sex, and $71.3 \%$ knew what to do in risk situations. Fourteen had had a test for HIV, 10 of whom had also reported having sexual relations. The level of knowledge was generally low and inconsistent. Conclusion: The results show that young people are at risk if contracting HIV/AIDS.

(Key words: HIV; adolescent health: health knowledge. altitudes, practice; safe sex. Paraguay). Pediatr. (Asunción), Vol. 33; N² 2; 2006
\end{abstract}

\section{RESUMEN}

Introducción: La generación actual de jóvenes es la mayor de la historia, casi la mitad de la población mundial tiene menos de 25 años. No han conocido un mundo sin SIDA. Los pocos países que han logrado disminuir su prevalencia nacional de VIH lo han hecho sobre todo inculcando comportamientos más seguros entre los jóvenes. Objetivos: Indagar los conocimientos, actitudes y prácticas sobre VIH/SIDA en 
jóvenes estudiantes de la educación media en colegios públicos y privados de Asunción y Lambaré, Paraguay. Material y Métodos: Investigación de diseño observacional. Analítico, prospectivo de corte transverso. Se aplicó una encuesta voluntaria, auto administrada, a estudiantes de $1^{\circ}, 2^{\circ}$ y $3^{\circ}$ de la media de colegios de la capital y Lambaré, durante el mes de agosto de 2006. Resultados: 336 encuestas analizadas. Edad media 16 años. 52\% masculinos, 48\% femeninos. La fuente de información citada con más frecuencia y la más importante fue la escuela. 42,4\% refieren relaciones sexuales, varones: 56,5\% y las mujeres en $27 \%$. La edad media de iniciación sexual de 14,6 años, 72\% de los varones se iniciaron sexualmente antes de los 15 años. 55\% refiere utilizar preservativo en relaciones ocasionales. 71,3\% sabía donde recurrir en situaciones de riesgo. Catorce se realizaron el test para VIH, 10 de los cuales habían referido relaciones sexuales. Nivel de conocimientos en general bajo e inconsistente. Conclusión: Los resultados revelan jóvenes en situación de riesgo de contraer VIH/SIDA.

(Palabras clave: VIH/SIDA, jóvenes, conocimientos, actitudes y prácticas, Paraguay).

Pediatr. (Asunción), Vol. 33; N 2; 2006

\section{Introducción}

Desde que los primeros casos del Síndrome de Inmunodeficiencia Adquirida (SIDA) se han reportado en 1981, la infección con el Virus de la Inmunodeficiencia Humana (VIH) ha crecido en proporciones pandémicas resultando en una estimación actual de 65 millones de infecciones y 25 millones de muertes. Durante el 2005 solamente, se ha estimado que 2,8 millones de personas murieron de SIDA, 4,1 millones de nuevos infectados y 38,6 millones viviendo con $\mathrm{VIH}^{1}$.

Un total de 39,5 millones de personas vivían con el VIH en el 2006, incluyendo los 3,4 millones de adultos y niños infectados por el VIH estimados en el mismo año, 400000 más que en 2004. Entre los de 15 años o más, los jóvenes representaron el $40 \%$ de las nuevas infecciones contraídas en el $2006^{2}$.

El crecimiento constante de la epidemia de SIDA se sustenta más que en las deficiencias de las estrategias de prevención, en la incapacidad del mundo para aplicar los instrumentos altamente eficaces de que dispone con miras a contener la propagación del VIH; muchos de los responsables de formular políticas se niegan a poner en marcha medidas cuya eficacia ha quedado demostrada. El hecho que el mundo no sea capaz de hacer llegar los métodos de prevención de reconocida eficacia a las personas que lo necesitan supone desaprovechar una gran oportunidad. Ampliar las estrategias de prevención disponibles en 125 países de ingresos bajos y medianos evitaría, según las estimaciones, 28 millones de nuevas infecciones por el VIH entre 2005 y 2015, más de la mitad de las nuevas infecciones que se prevé se produzca durante ese período, y representaría un ahorro de 24000 millones de dólares americanos, en gastos asociados al tratamiento. Combinando el tratamiento con esfuerzos efectivos de prevención se podría reducir dramáticamente los recursos necesarios para el tratamiento, en el largo plazo. Los esfuerzos para ampliar rápidamente y sostener el acceso al tratamiento antiretroviral se verán debilitados si no se rompe el ciclo de las nuevas infecciones por VIH. Cada vez se reconoce más que para ir por delante de la epidemia deben ampliarse e intensificarse los esfuerzos de prevención ${ }^{2-4}$.

Todas las estrategias deben reconocer que la prevención y el tratamiento del VIH están interrelacionados y que ambos deben acelerarse simultáneamente. El SIDA requiere una respuesta inteligente, rotunda y excepcional. Los esfuerzos descoordinados o los que ofrecen sólo soluciones parciales no reducirán significativamente el número de nuevas infecciones; disminuir y contener urgentemente la propagación de esta epidemia mundial requiere el acceso universal a la prevención, tratamiento y apoyo juntos. Si el mundo se moviliza de esta forma para ampliar simultánea y dinámicamente la prevención, tratamiento y atención del VIH, se podría lograr un auténtico criterio integral 
para abordar el SIDA, que frene la epidemia e invierta su curso ${ }^{5,6}$. Sin esta respuesta se prevé 45 millones de nuevos casos para el 2010. Los análisis de las intervenciones de prevención destinadas a estimular cambios en el comportamiento demuestran que dichos programas reducen la frecuencia de los comportamientos sexuales de riesgo ${ }^{3,7,8}$. Los programas orientados a inducir cambios de comportamiento suelen incluir información básica sobre el virus, evaluación del riesgo personal, asesoramiento del desarrollo de aptitudes tales como la capacidad para negociar el uso del preservativo con las parejas sexuales, acceso a preservativos y otras tecnologías de prevención. Estos programas constituyen las intervenciones de prevención más rentables y un elemento básico de todo programa nacional de prevención de VIH. Los programas públicos de educación y sensibilización dirigidos a la población general son fundamentales en toda respuesta firme al $\mathrm{VIH}^{3}$.

La generación actual de jóvenes es la mayor de la historia; casi la mitad de la población tiene menos de 25 años. No han conocido un mundo sin SIDA $^{9}$.

Los jóvenes de 15 a 24 años son los más amenazados -mundialmente representan la mitad de los nuevos casos de VIH- el futuro de la epidemia tomará forma a partir de los actos de esos jóvenes. Los pocos países que han logrado disminuir la prevalencia nacional del VIH lo han hecho sobre todo inculcando comportamientos más seguros entre los jóvenes. Una variedad de factores sitúa a los jóvenes en el centro de la vulnerabilidad al VIH. Entre ellos figuran la falta de información, educación y servicios sobre el VIH, los riesgos que muchos tienen que correr para sobrevivir, y los riesgos que acompañan a la experimentación y la curiosidad de los adolescentes. Riesgo elevado y vulnerabilidad elevada por inicio sexual precoz, las diferencias por razón de sexos, los consumidores jóvenes de drogas intravenosas corren un riesgo especial, porque pueden no tener el conocimiento ni las aptitudes para protegerse de la infección por un equipo de inyección contaminado ${ }^{10}$.

Brasil, fue uno de los países más tempranamente afectados por la epidemia de SIDA. Después del primer caso reportado en 1982, en
1990 se estimaba que Brasil tendría 1200000 casos de infección por VIH, sin embargo, una estimación reciente (2002) reduce a menos de 600000 los casos de infección. Los resultados de la respuesta del Brasil frente a la epidemia se pueden resumir en interacción entre estado y sociedad. De una manera importante la epidemia de VIH/SIDA en el Brasil ha contribuido a fortalecer sustancialmente las instituciones democráticas, a un mayor reconocimiento de los brasileños como ciudadanos, y a abrir un debate sobre la ética y la atención nacional a la salud ${ }^{11}$.

El conocimiento y la información son las primeras líneas de defensa para los jóvenes, sin embargo, el acceso a la educación sobre SIDA está lejos de ser universal, en América Latina y el Caribe sólo 4\% y 38\% de los jóvenes no escolarizados y escolarizados respectivamente tienen acceso a la educación sobre prevención. En la región la epidemia está bien consolidada y asume muchas facetas dada la diversidad en los patrones demográficos y de desarrollo de los países de la región, por lo cual existe el peligro que se propague de forma rápida en ausencia de respuestas nacionales eficaces ${ }^{12}$.

En Paraguay, unas 13000 (6 200-41 000) personas vivían con el VIH a final de 2005. Los varones constituyen la mayoría de los casos de SIDA, la exposición a equipos de inyección no estériles y las relaciones sexuales sin protección, entre varones, son los principales modos de transmisión del virus ${ }^{13}$.

Pocas fuentes proveen información acerca de VIH/SIDA en Paraguay. En 1992, una investigación prenatal a mujeres embarazadas no detectó infección por VIH. Por el 2001 la prevalencia en mujeres embarazadas estaba por debajo de 1\%. Estudios en trabajadores del sexo comercial en Asunción, entre 1987 y 1990, hallaron $0,1 \%$ de infectadas por VIH, y $0,17 \%$ de donantes de sangre, en un estudio no publicado del Banco Mundial en el 2001, esta misma vigilancia indicó que $1 \%$ de los exámenes voluntarios hechos en reclutas militares y 15\% de exámenes voluntarios en Usuarios de Drogas Intravenosas (UDIs) fueron positivos para el VIH. Estos números sugieren que la epidemia es más seria que lo que había sido reconocido y que está concentrada en UDIs más que 
en población general heterosexual y hombres que tienen sexo con hombre (HSH), como la estadística oficial lo indica. Paradójicamente, mientras solamente $15 \%$ de las mujeres de 15 a 45 años conocía dos o más vías para prevenir la infección por VIH, cerca del $80 \%$ en este grupo de edad reportaron haber usado condón durante su más reciente relación riesgosa ${ }^{14}$.

En Paraguay, se han notificado 1160 casos de SIDA desde 1986 hasta abril 2005. Están registrados 2856 VIH positivos. El crecimiento es constante, sin aceleraciones ${ }^{15}$.

La mayor proporción de personas afectadas tiene entre 25 y 49 años $(81,1 \%)$. La tendencia es el aumento de casos en las fajas etareas más jóvenes, con 32,2\% de los casos registrados entre los 15 y 24 años (un tercio del total). Los menores de 15 años registran el 14,65\% del total, para ambos sexos. La incidencia se mantiene creciente en forma sostenida, con predominio masculino, mayor en zonas urbanas, $y$ con afectación de personas pertenecientes a grupos etáreos jóvenes, en ambos sexos. La epidemia tiende a ser femenina, en población cada vez más joven, y a abarcar áreas geográficas del interior del país. El conocimiento general no revela pautas de prevención y protección firmemente establecidas ${ }^{15}$.

Se calcula en 20 mil los casos no registrados, sin embargo, se reconoce que podría haber un sub registro del 80\%, el más alto en América Latina $^{16,17}$.

En el país existe poca información sobre conocimientos, actitudes y prácticas de los jóvenes frente al VIH/SIDA, con la percepción que los programas de prevención no tienen la fuerza que debieran en el momento actual, se considera necesario investigaciones que permitan conocer el estado de los conocimientos, actitudes y prácticas en grupos vulnerables para definir políticas adecuadas que integren globalmente las áreas de prevención, tratamiento y atención al VIH/SIDA.

\section{Objetivo}

Indagar los conocimientos, actitudes y prácticas sobre el VIH/SIDA en jóvenes estudiantes del primero, segundo y tercer año de la educación media en colegios públicos y privados de la ciudad de Asunción y Lambaré.

\section{Pacientes y Métodos}

Diseño observacional, analítico, prospectivo, de corte transverso.

En horario de clases se aplicó un cuestionario auto administrado en forma anónima a estudiantes de la educación media en seis colegios capitalinos durante el mes de agosto del 2006.

Criterio de inclusión: alumnos que aceptaron responder la encuesta en forma anónima.

Criterio de exclusión: alumnos que no aceptaron responder el cuestionario, que no se encontraban en el momento de la toma de datos, y sujetos que no respondieron más de la mitad de los puntos contenidos en el cuestionario.

Instrumento: la encuesta se basó en un instrumento ya elaborado ${ }^{18}$ que adopta la modalidad de un cuestionario voluntario y anónimo, integrado por siete puntos, destinado a relevar información de diferentes niveles del conocimiento y de actitudes. En su estructura el cuestionario tiene el eje A destinado a establecer un perfil familiar y contextual del sujeto. La condición de anonimato posibilita respuestas más ajustadas a los hechos y al modo en que se piensa y se actúa.

El eje B interroga respecto de la "fuente" sobre la que se ha recibido información sobre VIH/SIDA. Se han propuesto quince posibilidades y la consigna se ha redactado de modo que se deben seleccionar cinco posibilidades siguiendo una jerarquía decreciente. Esta modalidad de "elección jerarquizada" permite, en el análisis posterior, establecer una ponderación de fuentes, destacando las vías fundamentales para la transmisión de la información y aquéllas que no resultan relevantes.

El eje C revela respecto de las posibilidades de prevención, las conductas preventivas efectivamente ejercidas y la etapa y el sujeto de iniciación genital.

El eje D indaga respecto a posibles representaciones del sujeto vinculadas con el VIH/ SIDA. Se ha propuesto en la consigna la selección de 5 posibilidades sobre 18 posibles. 
Esta modalidad de elección permitirá discriminar las representaciones significativas y descartar aquéllas que no lo son.

El eje E indaga respecto de una conducta eventual en el caso de una posible situación de riesgo, al tiempo que releva el nivel de conocimiento operativo del que disponen los sujetos "en situación".

El eje F indaga específicamente el comportamiento establecido respecto al uso del preservativo, más allá del nivel de conocimiento.

El eje G, está conformado por 22 afirmaciones, pondera la relevancia de cuatro categorías, en los acuerdos y desacuerdos de los sujetos.

Entrada de datos y procesamiento estadístico:

Los datos fueron almacenados en una base de datos informatizada (Epilnfo 6.0), y fueron sometidos a análisis descriptivo. Se realizó tabulación cruzada y pruebas de $\chi^{2}$. Se aceptó significativa la prueba con valor $\mathrm{p}<0,05 \mathrm{y}$ un nivel de confianza del 95\%.

\section{Consideraciones éticas}

A través de una nota se solicitó permiso a directivos del colegio asegurando el anonimato del mismo, y el compromiso de devolverles los resultados de la investigación y a través de una charla educativa dirigida a los estudiantes.

\section{Resultados}

Se distribuyeron 353 cuestionarios y se analizaron 336 cuestionarios administrados a jóvenes en 6 colegios. Diecisiete cuestionarios se desecharon por estar incompletos en más de la mitad de los ítems. Los diferentes (n) que se detallan a continuación, expresan que en algunas preguntas las respuestas estaban vacías.

\section{Perfil familiar y contextual}

Del total de la muestra correspondió a colegios privados ${ }^{2}$ el 29\% (98/336) y 71\% (238 7 336) a colegios públicos ${ }^{4}$. La frecuencia de participantes por cada colegio se observa en la figura 1.

La edad media fue de 16 años. Rango 14-25 años. La edad hasta 19 años fue el 95\% (316/ 336).
El 52\% (172/333) correspondió al sexo masculino y el 48\% (161/333) al femenino. El 70,4\% (235/334) refirió vivir con padre, madre y hermanos. Con la madre el 15\% (50/334), con el padre el 2,7\% (9/334); con la abuela el 1,5\% (5/ $334)$, con su pareja el 2,4\% (8/334) y otros el $8,1 \%(27 / 334)$.

Refirió ser soltero el 87\% (286/329); Casado el 1,8\% (6/329) y en pareja el 11,2\% (37/329).

\section{Fuentes de información}

El colegio fue la fuente de información más citada 179/336 y de éstos el 47\% (84/179) refirió en primer lugar en orden de importancia. La siguiente fuente de información más citada fue "Charla con amigos” 123/336 y en orden de importancia refirieron como primer lugar el 20,3\% (25/123); la TV 113/336 y de este grupo como fuente de información más importante lo refirió el 8,8\% (10/113) (figuras 2 y 3).

\section{Iniciación sexual y conductas preventivas}

El 42,4\% (139/328) dijo que ya tuvo relaciones sexuales; el 43,9\% (144/328) expresó no haber tenido relaciones sexuales y el 13,7\% (45/328) no quiso contestar. En relación al género los varones dijeron haber tenido relaciones en el 56,5\% (95/168) y las mujeres el 27\% (42/ 157) $\mathrm{p}<0,0000001$.

Dijeron tener relaciones sexuales el 30\% (29/96) de los alumnos de colegios privados y el 47\% (110/232) de los alumnos de colegios públicos $\mathrm{p}<0,01$.

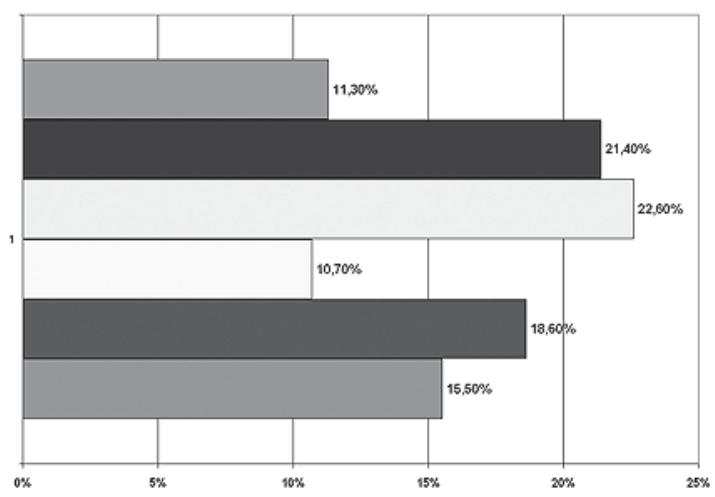

Figura 1. Distribución porcentual de los sujetos encuestados según colegio. 


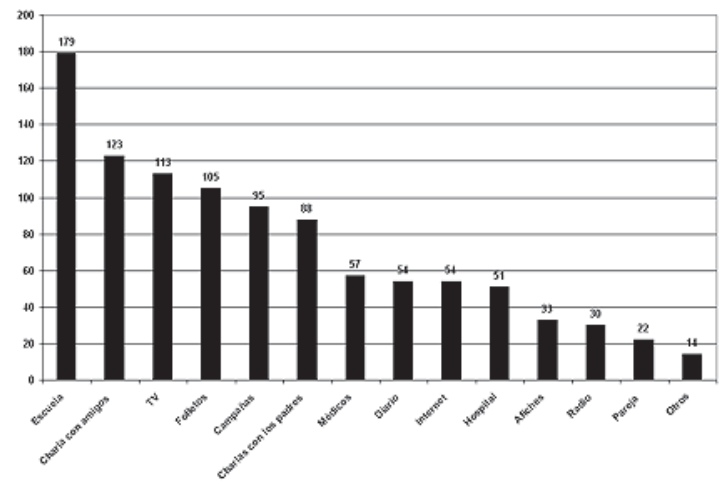

Figura 2. Frecuencia de la fuente sobre la que ha recibido información sobre VIH/SIDA.

Tabla 1. Edad de inicio de las relaciones sexuales en relación al género

\begin{tabular}{cccc}
\hline Edad de inicio & Masculino & Femenino & Total \\
\hline 9 & 2 & 0 & 2 \\
10 & 2 & 0 & 2 \\
11 & 1 & 0 & 1 \\
12 & 5 & 1 & 6 \\
13 & 10 & 3 & 13 \\
14 & 16 & 5 & 21 \\
15 & 19 & 8 & 27 \\
16 & 12 & 8 & 20 \\
17 & 4 & 1 & 5 \\
18 & 4 & 3 & 7 \\
20 & 1 & 0 & 1 \\
Total & 76 & 29 & 105 \\
\hline
\end{tabular}

La edad media de iniciación sexual fue 14,6 años, rango entre 9 y 20 años. Moda 15 años. La diferenciación por sexo se indica en la tabla 1.

Las primeras relaciones sexuales tuvo con el novio/a el 54,6\% (89/163); con amigo/a el 25,8\% (42/163); con un familiar el 4,9\% (8/163) y otro el 14,7\% (24/163).

$\mathrm{Al}$ indagar sobre los criterios que tiene en relación a la protección que ofrece el preservativo con el VIH las respuestas se dividieron de la siguiente manera: No protege expresó el 9,5\% (31/328); Protege el 16,5\% 54/328); No siempre protege el 56,7\% (186/328); No sabe el $17,4 \%(57 / 328)$.

"Usar preservativos en relaciones sexuales con personas conocidas ocasionalmente" las respuestas estuvieron expresadas de la siguien-

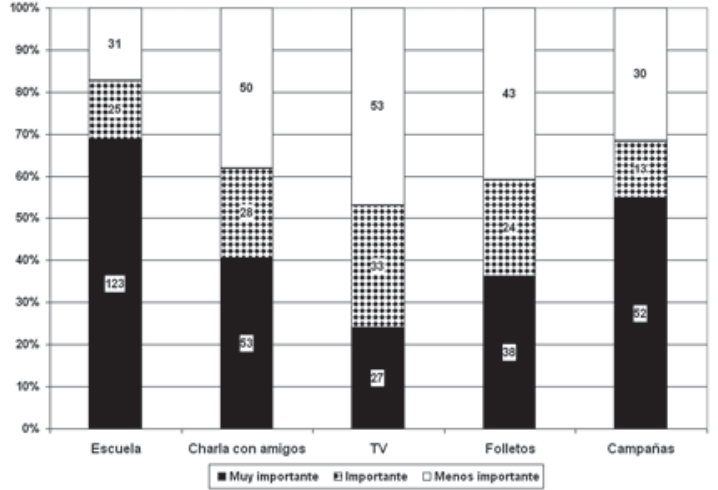

Figura 3. Frecuencia de las fuentes que dieron mayor información según orden de importancia.

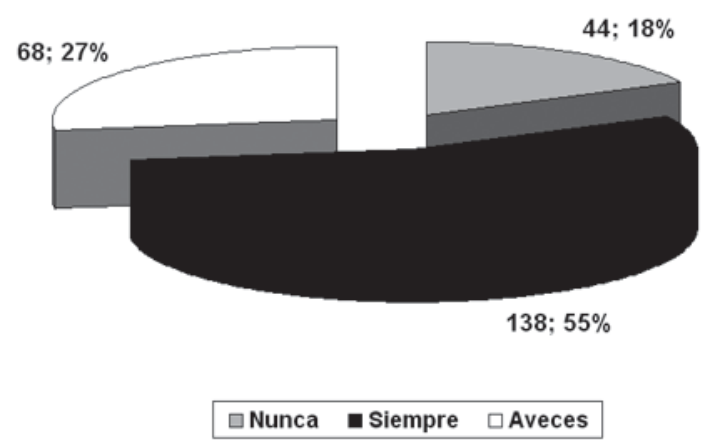

Figura 4. Frecuencia sobre las respuestas en relación al uso de preservativos.

te manera: No protege expresó el 17,4\% (24/ $325)$; protege el 28,3\% (92/325); No siempre protege el 42,8\% (139/325); no sabe el 21,5\% (70/325). Entre ambas preguntas, las respuestas presentan similitud y la asociación es estadísticamente significativa $\mathrm{p}<0,0000000$.

En la figura 4 se observa las respuestas que dieron en relación a la utilización de preservativos con personas conocidas ocasionalmente.

\section{Conducta ante una situación de riesgo}

No le ofrecieron hacer el test para el VIH al $91,7 \%$ (287/313) y si le ofrecieron al 8,3\% (26/ 313). El 4,5\% (14/313) dijo que se hizo el test para el VIH y nunca el 95,5\% (299/313). Figura 5.

De los sujetos que se hicieron el test para el 


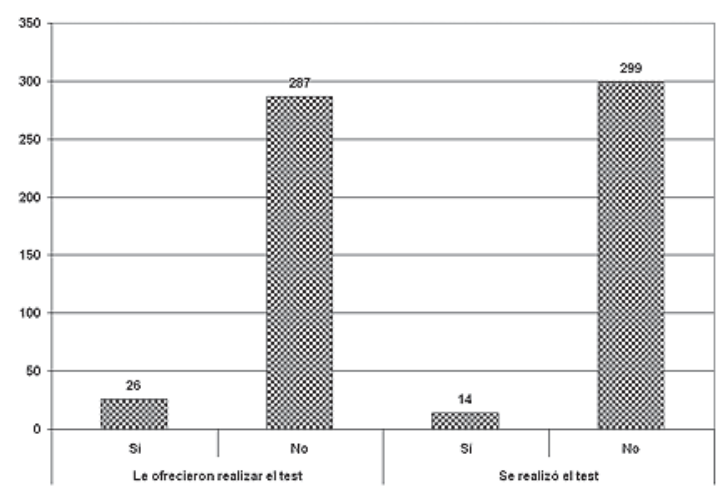

Figura 5. Diferencias entre el ofrecimiento para realizar el test y los que se hicieron el test para el VIH.

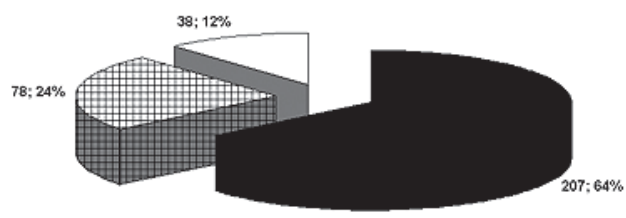

- Sabria donde acudir a No sabria donde acudir $\square$ Sabria donde acudir, pero no lo haria por vergũenza

Figura 6. Frecuencia de respuestas ante una situación de riesgo.
VIH 71\% (10/14) tuvieron relaciones sexuales, y el 28\% (4/14) de los que se hicieron el test para el VIH fueron sujetos que en sus respuestas prefirieron no contestar $\mathrm{p}<0,005$.

En la figura 6 se observa las respuestas dadas en caso de pasar por una situación de riesgo en la que fuera posible la exposición con el VIH.

\section{Comportamiento respecto al uso de preservativo, más allá del conocimiento}

Le cuesta plantearlo, pero prefiere tener la relación con preservativo y estar tranquilo después, 71,3\% (201/282); no sabe como decirlo $6 \%(17 / 282)$; sabe que debería usarlo pero no siempre lo hace el 14,5\% (41/282); y en el momento se olvida, y no piensa en eso el $8,2 \%$ $(23 / 282)$.

\section{Relevancia de los conocimientos, acuerdos y desacuerdos de los sujetos}

La ponderación de acuerdo a diferentes categorías (conocimiento de la enfermedad, vías de transmisión/ prevención, creencias o concepciones, nivel de información) expresada en acuerdos o desacuerdos de los sujetos se exponen en las tablas 2 a la 5 .

$\mathrm{Al}$ comparar la creencia con que se contaba con una vacuna eficiente los estudiantes del colegio privado lo afirmaron en un $16 \%(13 / 86)$ y los del colegio público en un 35\% (73/86) estas diferencias fueron significativas $\mathrm{p}<0,003$.

\section{Representaciones significativas}

Tabla 2. Ponderación del conocimiento de los sujetos en relación a la enfermedad

\begin{tabular}{|c|c|c|c|}
\hline Conocimiento de la enfermedad & $\begin{array}{l}\text { Afirmaron ser } \\
\text { Verdadera }\end{array}$ & $\begin{array}{l}\text { Afirmaron ser } \\
\text { Falsa }\end{array}$ & Total \\
\hline esperar a que se manifieste & $72 \quad(22 \%)$ & $(78 \%)$ & 328 \\
\hline $\begin{array}{l}\text { EI SIDA ataca el sistema inmunológico produciendo } \\
\text { enfermedades oportunistas }\end{array}$ & $248 \quad(83 \%)$ & $50 \quad(17 \%)$ & 298 \\
\hline $\begin{array}{l}\text { El SIDA es el resultado de la destrucción progresiva } \\
\text { de las defensas, causada por el virus }\end{array}$ & $276 \quad(85 \%)$ & $(14 \%)$ & 321 \\
\hline $\begin{array}{l}\text { El SIDA puede producir infecciones graves y } \\
\text { desarrollar cáncer }\end{array}$ & 196 (64\%) & 111 (36\%) & 307 \\
\hline $\begin{array}{l}\text { La enfermedad no se cura pero existen tratamientos } \\
\text { para controlarlas }\end{array}$ & 307 (96\%) & $(4 \%)$ & 321 \\
\hline $\begin{array}{l}\text { Los síntomas del SIDA pueden aparecer después } \\
\text { de algunos meses hasta algunos años }\end{array}$ & $291 \quad(91 \%)$ & $(9 \%)$ & 320 \\
\hline
\end{tabular}


Tabla 3. Ponderación sobre prevención y vías de transmisión del SIDA

\begin{tabular}{|c|c|c|c|}
\hline Prevención y vías de transmisión & $\begin{array}{l}\text { Afirmaron ser } \\
\text { Verdadera }\end{array}$ & $\begin{array}{l}\text { Afirmaron ser } \\
\text { Falsa }\end{array}$ & Total \\
\hline el embarazo, el parto y lactancia & 266 (82\%) & $(18 \%)$ & 324 \\
\hline $\begin{array}{l}\text { EI VIH se puede transmitir por transfusión de } \\
\text { sangre contaminada }\end{array}$ & $(94 \%)$ & $(6 \%)$ & 329 \\
\hline Un beso profundo puede transmitir el VIH & 125 (38\%) & 205 (62\%) & 330 \\
\hline $\begin{array}{l}\text { Los contactos buco-genitales (sexo oral) pueden } \\
\text { ser vía de transmisión del VIH }\end{array}$ & $240 \quad(77 \%)$ & $72 \quad(23 \%)$ & 312 \\
\hline El virus VIH se puede transmitir por vía sexual & 312 (97\%) & $(3 \%)$ & 323 \\
\hline $\begin{array}{l}\text { El virus VIH se puede transmitir por compartir } \\
\text { agujas, jeringas y accesorios contaminados }\end{array}$ & $311(97,5 \%)$ & $8 \quad(2,5 \%)$ & 319 \\
\hline
\end{tabular}

Tabla 4. Ponderación sobre creencias o concepciones

\begin{tabular}{lrrrr}
\hline Creencias o concepciones & $\begin{array}{c}\text { Afirmaron ser } \\
\text { Verdadera }\end{array}$ & $\begin{array}{c}\text { Afirmaron ser } \\
\text { Falsa }\end{array}$ & Total \\
\hline $\begin{array}{l}\text { Nadie puede impedir la transmisión del VIH, } \\
\text { depende del destino y suerte de cada uno }\end{array}$ & $119 \quad(36 \%)$ & $210 \quad(64 \%)$ & 329 \\
$\begin{array}{l}\text { La única manera de saber si contraje el VIH es } \\
\text { esperar a que se manifieste }\end{array}$ & 72 & $(22 \%)$ & $256 \quad(78 \%)$ & 328 \\
$\begin{array}{l}\text { Frecuentar una piscina puede ser factor de riesgo } \\
\text { para la infección del VIH }\end{array}$ & $59 \quad(19 \%)$ & $255 \quad(81 \%)$ & 314 \\
$\begin{array}{l}\text { En la actualidad no existen tratamientos para } \\
\text { frenar la enfermedad }\end{array}$ & $180 \quad(58 \%)$ & $129 \quad(42 \%)$ & 309 \\
$\begin{array}{l}\text { Compartir un baño de personas desconocidas } \\
\text { puede ser un riesgo para la infección del VIH }\end{array}$ & $116 \quad(37 \%)$ & 195 & $(63 \%)$ \\
$\begin{array}{l}\text { Besar y abrazar a una persona infectado con el } \\
\text { VIH es una vía de transmisión }\end{array}$ & $33 \quad(10 \%)$ & $289 \quad(90 \%)$ & 311 \\
\begin{tabular}{l} 
Por suerte se cuenta con una vacuna eficiente \\
\hline
\end{tabular} & 86 & $(30 \%)$ & $203 \quad(70 \%)$ & 322 \\
\hline
\end{tabular}

Tabla 5. Ponderación sobre nivel de información

\begin{tabular}{lcccc}
\hline Nivel de información & $\begin{array}{c}\text { Afirmaron ser } \\
\text { Verdadera }\end{array}$ & $\begin{array}{c}\text { Afirmaron ser } \\
\text { Falsa }\end{array}$ & Total \\
\hline $\begin{array}{l}\text { Los resultados de un análisis de VIH son confi- } \\
\text { denciales }\end{array}$ & $225 \quad(72 \%)$ & $86 \quad(28 \%)$ & 311 \\
$\begin{array}{l}\text { No existe análisis de VIH que se realice de forma } \\
\text { gratuita }\end{array}$ & $143 \quad(47 \%)$ & $163 \quad(53 \%)$ & 306 \\
$\begin{array}{l}\text { La seropositividad para el VIH significa tener la } \\
\text { enfermedad del SIDA }\end{array}$ & 141 & $(51 \%)$ & 135 & $(49 \%)$ \\
$\begin{array}{l}\text { Una manera de saber si tengo VIH es hacerme } \\
\text { un análisis especial en un centro de salud }\end{array}$ & $307 \quad(96 \%)$ & 14 & $(4 \%)$ \\
\hline
\end{tabular}




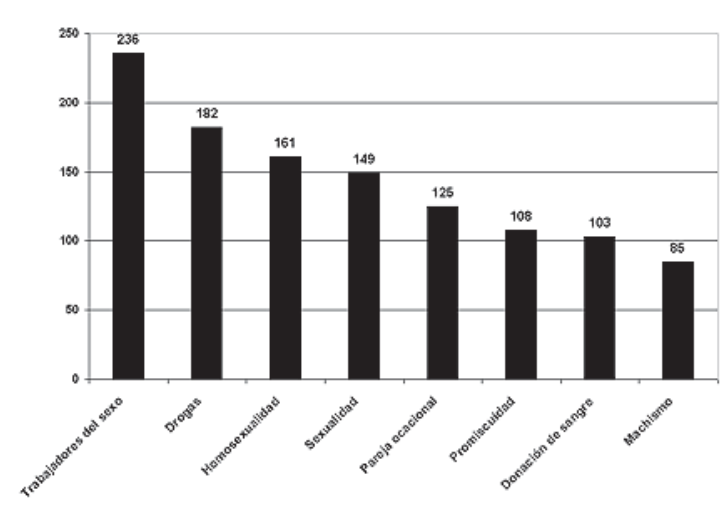

Figura 7. Frecuencia de las palabras que más relacionaron con el VIH/SIDA.

En la figura 7 se observa las palabras que más relacionaron con el VIH/SIDA. De las 18 opciones que tuvieron sólo se muestra las más nombradas.

\section{Discusión}

En países donde el VIH está concentrado entre trabajadores del sexo, usuarios de drogas intravenosas, hombres que tienen sexo con hombres, como sucede en el Paraguay, las conductas de riesgo comienzan durante la adolescencia, y gran proporción de esta población de riesgo son menores de 25 años. En países con epidemias generalizadas también los jóvenes se hallan en el centro de las mismas ${ }^{19}$.

La vasta mayoría de los mismos permanece sin información acerca del sexo y de las infecciones transmitidas sexualmente (ITS). Aunque una mayoría ha oído hablar del SIDA muchos no saben como se expande y no creen estar en riesgo. Aquellos jóvenes que conocen algo acerca del VIH a menudo no se protegen a sí mismos pues carecen de habilidades o medios para adoptar conductas seguras ${ }^{19,20}$.

En América Latina y el Caribe la población entre los 10 y 24 años de edad alcanzó 155 millones en el año 2000, lo cual representa alrededor de un tercio de la población total de la región. En esta región muchos jóvenes están teniendo relaciones sexuales, a menudo sin protección, lo cual los coloca en riesgo de embarazos no deseados, abortos inseguros, e infección con enfermedades de transmisión sexual, incluyendo el $\mathrm{VIH}^{21}$.
Retrasando la edad de inicio de relaciones sexuales los jóvenes pueden protegerse de la infección. Los adolescentes que inician su actividad sexual a edad temprana tiene mayor riesgo de infectarse con el VIH; investigaciones en diferentes países ha mostrado que los adolescentes que inician su actividad sexual muy jóvenes tienen mayor probabilidad de tener sexo con parejas con elevado riesgo, múltiples parejas, y menor probabilidad de utilizar condones ${ }^{19}$.

En América Latina y el Caribe la edad de la primera relación sexual es significativamente más baja para los hombres que para las mujeres. En general la experiencia sexual se inicia antes en los varones que en las mujeres, con un cuarto a tres cuarto de los varones iniciando sus relaciones sexuales antes de los 15 años $^{22}$.

En la CAPSR'02 (Conocimientos, Actitudes y Prácticas en Salud Reproductiva) en Adolescentes no escolarizados de zonas vulnerables del Área Metropolitana, Asunción, Paraguay, 2002 que incluyó adolescentes femeninos y masculinos de 12 a 19 años de edad (universo: 320 ), se reporta que el 45,9\% había iniciado vida sexual activa. La edad de inicio entre los 8 y los 13 años fue en 30\% de los encuestados, de estos el 8,2\% lo hizo antes de los 11 años, $58,3 \%$ fueron varones y $41,7 \%$ mujeres. El $71 \%$ de todos los encuestados manifestaron haberse iniciado sexualmente antes de los 15 años esto es igual a 7 de cada 10 adolescentes quienes tuvieron su primera relación coital antes de los 15 años ${ }^{23}$.

En esta investigación se dan resultados similares. La edad media de inicio fue de 14,6 años, con rango de 9 a 20 años. El 57\% de los varones y $27 \%$ de las mujeres afirmaron tener relaciones sexuales, $30 \%$ de los alumnos de colegios privados y el $47 \%$ de los estudiantes de colegios públicos. Los varones iniciaron las relaciones sexuales más temprano que las mujeres. El 72\% de los varones que refieren relaciones sexuales las iniciaron a los 15 años o antes. El riesgo de infección por el VIH entre los jóvenes se halla directamente relacionado con la edad de inicio de la actividad sexual. Estos hallazgos los sitúan en riesgo para la adquisición de infecciones de transmisión sexual, entre ellas VIH/SIDA.

El conocimiento y la información son las 
primeras líneas de defensa para los jóvenes, algunos países han adoptado medidas atrevidas para abordar las necesidades de información sobre VIH/SIDA, pero esta educación sigue estando lejos de ser universal. En África Subsahariana sólo el 8\% de los jóvenes no escolarizados y un número ligeramente superior de jóvenes escolarizados tiene acceso a la educación sobre prevención. Las cifras equivalentes para Europa Oriental y Asia Central son de $3 \%$ para los jóvenes no escolarizados y del $40 \%$ para los escolarizados, mientras que en el Caribe y América Latina son del 4 y 38\% respectivamente. Un estudio global demostró que 44 de los 107 países no incluían el SIDA en los programas escolares.

No es de extrañar que los datos de 20 países con alta prevalencia revelen que a pesar que la mayoría de los jóvenes ha oído hablar sobre VIH/SIDA, la mayor parte es incapaz de reconocer tres ideas erróneas acerca de VIH ni de identificar dos métodos de prevención ${ }^{23,24}$.

La presente investigación revela que existe un nivel de comprensión superficial y de manera general, una cierta, desinformación conceptual.

En la ponderación sobre creencias o concepciones se ha hallado porcentajes muy elevados de conocimientos inadecuados.

Con relación a la prevención y vías de transmisión, se ha detectado un elevado nivel de conocimiento con respecto a la transmisión sexual y a través de jeringas (97\%), y por vía de transfusión (94\%). Por otro lado, un porcentaje elevado que va del 23 al 38\% ignora otras vías de contagio como la del contacto bucogenital, la transmisión vertical, y afirman en un $38 \%$ que el beso profundo puede transmitir el VIH. Con estos datos se concluye que los conocimientos no tienen consistencia, y debe llamar la atención sobre los contenidos de los programas educativos formales pues estos conocimientos científicamente comprobados deben transmitirse fundamentalmente en el ámbito escolar.

La presencia de la escuela como promotora de la educación sexual se evidencia en esta investigación como la fuente de información citada con mayor frecuencia y en primer lugar, seguida por charla con amigos y la televisión.
En las estrategias de educación volcadas a la educación sexual en los adolescentes es importante considerar su red de relaciones y abarcar no solamente profesores, grupos de pares, profesionales de la salud como fuentes de información y diálogo, sino también los padres, las madres y otros miembros de la familia. Los profesionales de la salud y los profesores deberían ser capacitados para que sus intervenciones no se centren en el modelo biológico y puedan iniciar discusiones, estimular reflexiones acerca de la sexualidad en una dimensión socialmente construida contemplando sus perspectivas físicas, psicológicas, emocionales, culturales y sociales, evitando un reduccionismo biológico con la intención de una promoción integral de la salud del adolescente ${ }^{25}$.

Los medios de comunicación desempeñan un papel importante en el fomento de la sensibilidad y la difusión de conocimientos acerca del VIH, y por consiguiente, en la prevención de la infección ${ }^{13}$. Los medios de comunicación son cada vez más importantes en la vida de los jóvenes. En muchos países representan causes excelentes a través de los cuales se difunden mensajes de prevención de VIH. La investigación ha demostrado que las campañas de los medios de comunicación son más eficaces cuando se combinan con esfuerzos de educación locales ${ }^{24}$.

El uso del preservativo es el indicador más confiable para definir el perfil de percepción del riesgo del VIH en la población sexualmente activa $^{26}$.

Con respecto al comportamiento del uso del condón en la investigación actual el 71,3\% prefiere tener relación sexual con preservativo y estar tranquilo después. De los que dijeron tener relaciones sexuales sólo lo utiliza el 55,8\% en caso de relaciones ocasionales.

No lo utilizan de modo sistemático en todas sus relaciones sexuales, poniendo de manifiesto la vulnerabilidad del grupo en cuanto al riesgo de infección con VIH. La mayoría de los expertos en salud de los adolescentes está de acuerdo en que los programas integrales de prevención del VIH -que promueven paralelamente el uso del preservativo y el retraso del inicio de la actividad sexual- entre quienes son sexualmente activos, constituyen el modo más 
eficaz de abordar la prevención del VIH destinada a los jóvenes ${ }^{2}$.

En el Brasil, reconocido por el tratamiento para el SIDA patrocinado por el gobierno para todo el país, los esfuerzos persistentes y agresivos para prevenir nuevos casos de infección han jugado un rol igual o mayor en la disminución de la expansión del virus. Campañas promoviendo el uso del condón tanto para los grupos de riesgo como para la población general ha ayudado a mantener estable en un $0,6 \%$ la frecuencia de VIH entre los brasileños de 15 a 49 años desde el año $2000^{27}$. "Nosotros estamos tratando en gran escala, pero estamos haciendo concomitantemente prevención a gran escala, debido a que lo uno sin lo otro no tiene sentido"28.

Está visto que es importante y posible promover la monogamia y la reducción de parejas, junto a la abstinencia y el uso de condones. La gente es capaz de comprender que la raíz del problema con la transmisión de VIH es el sexo peligroso y adopta las conductas que mejor se ciñen a sus circunstancias, por ello es responsabilidad pública ayudar a la gente a comprender las fortalezas y limitaciones de cada uno de los componentes, en estrategias de prevención, y no promover uno en detrimento de otro. Estudios prospectivos han mostrado que el uso de condones reduce el riesgo de contagio en 80$90 \%$ cuando se usa siempre, en la vida real son usados a menudo incorrecta o inconsistentemente, por ello, su uso no debería ser promovido de manera que lleve a excesos de confianza o a conductas de riesgo ${ }^{29}$.

El retraso en el inicio de las relaciones sexuales en los jóvenes puede protegerlos significativamente de la infección por VIH. En áreas donde la infección por VIH está declinando es primariamente debido a que los jóvenes adolescentes reciben las herramientas e incentivos para adoptar conductas más seguras. Los jóvenes han demostrado que ellos son capaces de tomar decisiones responsables y protegerse a sí mismos y que pueden educar y motivar a otros. Para que los programas de cuidado y prevención sean efectivos los jóvenes deben ser comprometidos en el diseño e implementación de los mismos. La retención de los niños en las escuelas ayuda a la protección de los mismos contra la infección por VIH. La adolescencia temprana en edades de 10 a 14 años, es un tiempo en el que los patrones permanentes de conductas saludables pueden ser establecidos, como posponer el inicio sexual que puede mitigar la expansión de VIH/SIDA. Establecer patrones saludables desde el inicio es más fácil que cambiar conductas riesgosas ya establecidas. Los padres, las familias extendidas, la comunidad, las escuelas, los pares, son claves en guiar y sostener a los jóvenes para tomar decisiones seguras acerca de su salud y bienestar. Los estudios han demostrado que una conexión emocional consistente y positiva con un adulto protector ayuda a los jóvenes a sentirse a salvo y seguros $^{21}$.

Los gobernantes pueden contener la epidemia a costo relativamente bajo, invirtiendo en la prevención antes que VIH/SIDA llegue a ser un problema significativo de salud, proveyendo a la gente, en especial aquellas con alto riesgo de contraer infección por VIH, con la información y el soporte que necesitan para prevenir la infección. La prevención del VIH, al igual que el tratamiento es para toda la vida. En lugar de iniciativas de prevención aisladas o de corto plazo, los programas nacionales eficaces necesitan mantener a largo plazo las acciones de política y programáticas fundamentales a una escala suficiente, adaptarlas a medida que la epidemia evoluciona, y responder a los cambios que se produzcan en las características de la infección y los entornos sociales ${ }^{2,20}$.

Existe escasa información sobre la epidemia en el Paraguay. Considerada en sus inicios baja, la epidemia se ha transformado en una de prevalencia intermedia en poblaciones de mayor vulnerabilidad. Urge mayor información sobre el estado actual y acciones de prevención que sitúe a los jóvenes en el centro de las mismas, independientemente que las epidemias estén concentradas en grupos de alto riego.

En esta investigación, realizada en jóvenes estudiantes mediante una encuesta auto administrada que se halla sujeta a la honestidad de las respuestas y a la comprensión de las preguntas, se revela un elevado porcentaje de iniciación sexual temprana. La escuela es la fuente de información más importante sobre VIH/ SIDA. Las conductas preventivas, uso de con- 
dón, se da sólo en alrededor del 50\%. El nivel de conocimientos demuestra comprensión superficial y desinformación conceptual

\section{Conclusión}

En esta investigación los jóvenes muestran escasos conocimientos, prácticas inadecuadas de prevención y por consiguiente riesgo de adquirir la infección por VIH.

\section{Referencias}

1.- $\quad$ CDC: The Global HIV/A1DS Pandemic, 2006. MMWR. 2006; 55: 841-4.

2.- ONUSIDA: Prevención integral del VIH. Informe sobre la Epidemia de SIDA. Ginebra: ONUSIDA: 2006.

3.- Joint United Nations Programme on HIV/AIDS (UNAIDS). 2006 report on the global A1DS epidemic. Geneva, Switzerland: UNAIDS: 2006.

4.- Salomón JA, Hogan DR, Stover J, Stanecki KA, Walkern, Ghys PD, et al: Integrating H1V Prevention and Treatment: From Slogans to Impact. Plos Med 2005; 2 (1): e 16.

5.- ONUSIDA. OMS: Situación de la epidemia de SIDA. Diciembre 2005. Intensificar la prevención: un camino al acceso universal. Ginebra: OMS; 2005.

6.- Stover J, Walker N, Garnett GP, Salomón JA, Stanecki $K A$, Ghys PD, et al: Can we reverse the HIV/ A1DS pandemic with an expanded response? Lancet 2002; 360 (9326): 73-7.

7.- UNAIDS: Policy Prqject (2004): Coverage of selected services for HIV/AIDS prevention and care in low and middle-income countries in 2003. Washington. DC: UNAIDS: 2003.

8.- Crepaz N: Meta analysis of high risk sexual behaviors in person aware and anaware they are infected with VIH in the Unites State. Implications HIV pre-vention programs. J AIDS 2005; 39 (4): 446-53.

9.- UNFPA: Adolescents Fact Sheet. 2006. Washington: UNFPA: 2006.

10.- ONUSIDA: Informe sobre la epidemia mundial de SIDA, 2003. Ginebra: ONUSIDA: 2003.

11.- Levi G, Vitoria M: Fighting against AIDS: The Brazilian experience. AIDS 2002; (16): 2373-83.

12.- Estrada JH: Modelos de Prevención en la Lucha contra el VIH/SIDA. Acta Biohetica 2006; 12 (1): 91100.

13.- ONUSIDA: Situación de la Epidemia. Diciembre 2006. Ginebra: ONUSIDA: 2006.

14.- García-Abreu A, Noguer I, Cowgill K: Health. Nutrition and Population (HNP) discussion Paper. HIV/
AIDS in Latin America. The Challenges Ahead. Washington DC: The World Bank; 2003.

15.- Departamento de Vigilancia Epidemiológica. PRONASIDA: Programa Nacional del SIDA. Asunción: PRONASIDA: 2005.

16.- Agua Buena. Asociación de Derechos Humanos. Paraguay: Llamado a ONUSIDA, continúan las denuncias por desabastecimiento. San José de Costa Rica: Asociación de Derechos Humanos: 2004. Disponible en http://www.aguabuena.org/articulos/paraguay 20041205.html

17.- CEPEP: Encuesta Nacional de Demografía y Salud Sexual y Reproductiva - ENDSSR. Asunción: CE-PEP: 2004.

18.- Rasseto $M$, Herrera $M$, Massa $M$, Ruiz A: Diseño de un cuestionario para identificar actitudes sobre SIDA. Enseñanza de las Ciencias. Revista de Investigación y Experiencia Didáctica 2005; 23 (Supl): 1-7.

19.- UNAIDS/WHO: Preventing HIV/AIDS in young people: a systematic review of the evidence from developing countries. Geneva: WHO; 2006.

20.- World Health Organization: Young people and HIV/ AIDS opportunity in crisis. New York: UNICEF; 2002.

21.- Moya C: La Salud Sexual y Reproductiva en Jóvenes de América Latina y el Caribe. Washington: Advócales for Youth: 2002. Disponible en: http://www.vadvocates foryouth.org/publications/ factsheet/fssaludsexual/pdf.

22.- Lundgren R: Protocolos de Investigación para el Estudio de la Salud Sexual y Reproductiva de los adolescentes y jóvenes Varones en América Latina. Washington, DC: Organización Panamericana de la Salud: 2000.

23.- PROMESA-USAID-ICA: Conocimientos, actitudes y prácticas en Salud Reproductiva en adolescentes no escolarizados de zonas más vulnerables del Área Metropolitana. CAPSR’02. Asunción: USAID; 2002.

24.- ONUSIDA: Informe mundial sobre la epidemia de SIDA 2004: el VIH y los jóvenes: la amenaza para la juventud de hoy. Ginebra: ONUSIDA; 2004.

25.- Borges AL, Izumi Nichiata LY, Schor N: Conversando sobre sexo: a rede sociofamiliar como base de promocao da saúde sexual e reprodutiva de adolescentes. Rev Latino Am Enfermagem 2006; 14 (3): 314-8.

26.- Guchin Mieres M, Mere Rouco JJ: Jóvenes, sexualidad y VIH/SIDA en Uruguay Conocer para Prevenir. Montevideo: UNESCO: 2004.

27.- Bridges E: HIV Prevention in Developing Countries. Washignton: Advócales for Youth: 2006. Disponible en: http://www.advocatesforyoulh.org/publications/ faclsheel/fshivcounlries.hlm

28.- Okie S: Fighting HIV- lessons from Brasil. N Engl J Med 2006, 354 (19): 1977-81.

29.- Shellon J, Halperin D, Nantulya V, Polts M, Gayle H, Holmes K: Partner reductions is crucial for balanced "ABC" approach lo HIV prevention. BMJ 2004; 328: 891-3. 